\title{
Gastric actinomycosis: an rare endoscopic diagnosis
}

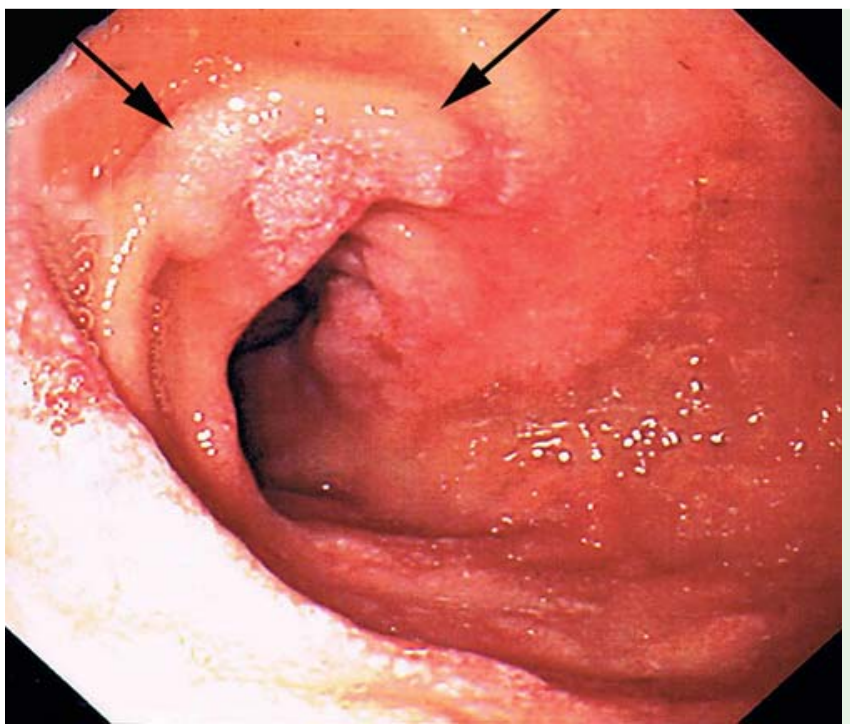

Fig. 1 Endoscopic appearance of the irregular ulcer on the greater curve of the stomach (arrow).

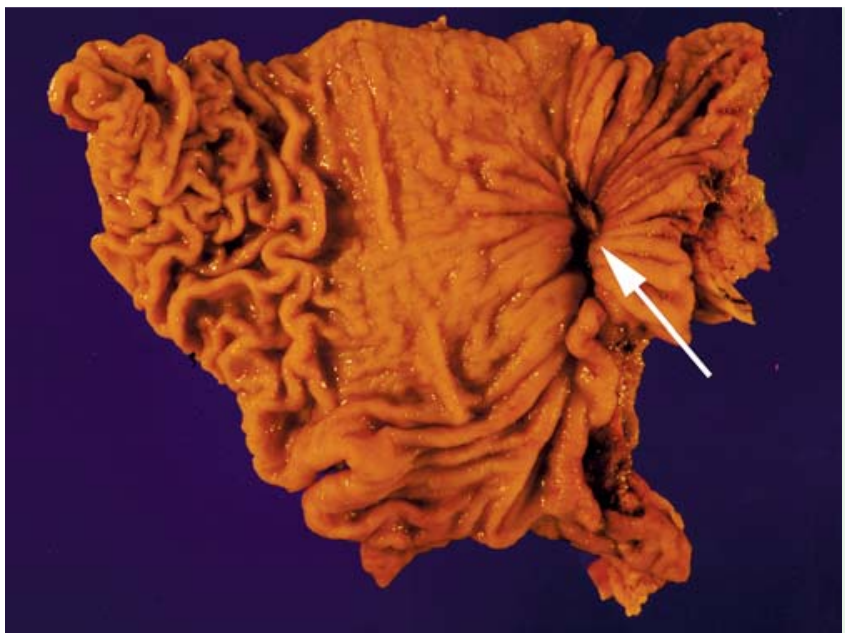

Fig. 3 Gross view of the surgically resected gastric mucosa depicting the ulcer (arrow).

A 67-year-old man presented to the emergency department with haematemesis. He had no past medical history and no history of anticoagulant, excess alcohol, steroid, or nonsteroidal anti-inflammatory medication use. He was haemodynamically stable, and on examination there were no stigmata of chronic liver disease but there was mild epigastric tenderness. Laboratory tests were normal except for a hemoglobin $(\mathrm{Hb})$ of $9.3 \mathrm{~g} / \mathrm{dL}$.

A diagnostic esophagogastroduodenoscopy (EGD) was performed, which revealed a large, irregular, malignant appearing ulcer on the greater curve of the stomach $(\bullet$ Fig. 1$)$.
Histological examination of the gastric ulcer samples obtained endoscopically showed chronic Helicobacter pylori-associated gastritis and severe mucosal ulceration without dysplasia. A computed tomography (CT) scan revealed irregularity and thickening of the posterior wall of the gastric mucosa suggestive of a malignant localized perforation. A repeat EGD was performed given the high index of suspicion of malignancy on the radiological and endoscopic findings. Endoscopically, the ulcer was the same with no evidence of healing. There were also similar histological findings on examination of the samples from the second EGD as the first, however,
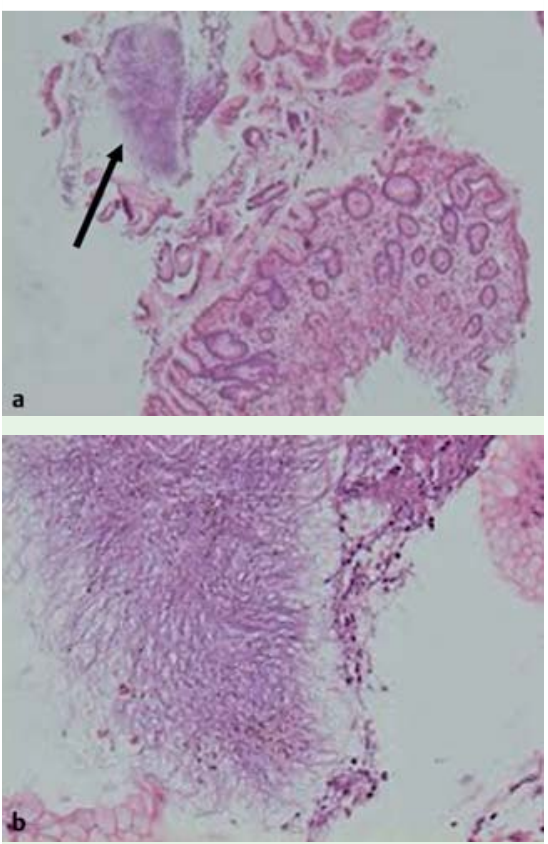

Fig. 2 a Hematoxylin and eosin stained histological section of the gastric ulcer showing the colony of Actinomyces (arrowed). b Higher power microscopy of the histological sample showing heavy Actinomyces growth.

in addition large colonies of Actinomyces were seen in the gastric mucosa ( $\bullet$ Fig. 2 ). The patient was treated with an extended course of oral penicillin. However, due to the suspiciously malignant appearance of the ulcer, he underwent resection (๑ Fig. 3).

Histological examination of the surgically resected specimens did not show evidence of malignancy but confirmed the presence of $H$. pylori gastritis and actinomycosis.

Most documented cases in the literature result from surgical procedures for presumed gastric malignancy [1-5]. To our knowledge, there are only two published case reports with endoscopically obtained biopsies [1,5]. Gastric actinomycosis is an extremely rare, chronic suppurative infection, rarely found in the upper gastrointestinal tract. We hope that our case increases awareness of this curable condition.

\section{Competing interests: None}

Endoscopy_UCTN_Code_CCL_1AB_2AD_3AC 
N. Patel ${ }^{1}$, H. Woodcock ${ }^{2}$, K. Patel ${ }^{2}$, B. Amo-Takyi ${ }^{2}$, A. Wotherspoon ${ }^{3}$, J. Thompson ${ }^{2}$, P. Prasad ${ }^{2}$

1 West Middlesex Hospital NHS Trust, Isleworth, UK

2 Chelsea and Westminster Hospital NHS Trust, London, UK

3 Royal Marsden Hospital, Fulham Road, London, UK

\section{References}

1 Lee SH, Kim HJ, Kim HJ et al. Primary gastric actinomycosis diagnosed by endoscopic biopsy: case report. Gastrointest Endosc 2004; 59: $586-589$

2 Van Olmen G, Larmuseau MF, Geboes K et al. Primary gastric actinomycosis: A case report and review of the literature. Am J Gastroenterol 2008; 79: 512-516

3 Rushrash G, Rasem G, Amar M, Rabinson S. Actinomycosis mimicking abdominal neoplasm. Haerfuah 1992; 122: 638 -639, 687

4 Wilson E. Abdominal actinomycosis, with special reference to the stomach. Br J Surg 1961; 49: 266-270

5 Öksüz M, Sandikci S, Culhaci A et al. Primary gastric actinomycosis: A case report. Turk J Gastroenterol 2007; 18: 44-46
Bibliography

DOI $10.1055 / \mathrm{s}-0030-1255709$

Endoscopy 2010; 42: E218-E219

(c) Georg Thieme Verlag KG Stuttgart · New York . ISSN 0013-726X

\section{Corresponding author}

\section{N. Patel}

West Middlesex Hospital

Isleworth

Twickenham TW7 6AF

nishakpatel1@gmail.com 